\title{
Summary of Evaluating Inertial Gyro Storage Life Based on Accelerated Life Test
}

\author{
Huizhi1 $\mathrm{Li}^{1, a}$, Shaoguang Wang ${ }^{2, \mathrm{~b}}$, Chen Huang ${ }^{1, \mathrm{c}}$ and Xihui Mu ${ }^{2, \mathrm{~d}}$ \\ ${ }^{1}$ Army Engineering University of PLA, Shijiazhuang 050000, China; \\ 2Special Service Institute, Shijiazhuang 050000, China. \\ a1452216774@qq.com, btiger7558841@163.com, c13272172420@163.com, dmxhvip@163.com
}

Keywords: inertial gyro; accelerated life test; life assessment Introduction

\begin{abstract}
In this paper, the life evaluation of inertial gyro components of information-based ammunition control system is carried out by using accelerated life test. The present research situation of accelerated life test technology is discussed, and the basic principle, shortcomings, the general process of evaluating the life of the inertial gyro components of the control system by the acceleration test and some problems needing attention in the life assessment process are put forward.
\end{abstract}

\section{Introduction}

Informational ammunition is a new type of ammunition that integrates information technology. It utilizes precise guidance system and relies on commonly used projectiles to search, identify and track targets from complicated background through information technology to guide and manipulate projectile Line, hit and destroy the target [1]. The control system of informational ammunition is the "brain and central nervous system" of informational ammunition, which combines a great deal of high-tech and materials with much higher complexity than ordinary ammunition. The most important navigation device in the control system is the inertial gyroscope, which is a high-precision instrument used to measure the vertical axis inclination of the projectile in the vertical plane of the trajectory and generate control signals in the inertial guidance section and the automatic guidance section. The accuracy and reliability have a decisive effect on the level and effectiveness of information munitions control systems. When evaluating the lifetime of an inertial gyroscope, the traditional method is based on monitoring the natural environment test life assessment method, but this method exists significant drawbacks, such as the need for a sufficient number of samples, which will result in greater financial and material resources, and the assessment period required for inertial gyroscopes using this method will be longer and apparently no longer suitable for contemporary mechanical and electrical products fast update speed. In [2], the reliability of optical fiber inertial gyroscope mainly depends on the performance degradation, fault tree and other aspects of the study. In reference, [3] as the structure of uncomplicated inertial gyroscope, it is proposed to evaluate the reliability of performance by extrapolation of parametric performance. In this paper, the life span of inertial gyroscopes will be briefly discussed through the method of accelerated life test. This method is of theoretical feasibility and can significantly improve the cost-effectiveness ratio of inertial gyroscope life assessment, which has some practical significance.

\section{The Current Situation of Life Assessment Based on Accelerated Life Test}

\subsection{The Basic Concepts Related to Life Assessment.}

In the ammunition system, a very critical tactical indicator is the storage life, so the assessment of the ammunition shelf life is a crucial task. The ammunition storage life assessment technology has been rapidly developed in the world [4].

The evaluation of storage life consists mainly of two parts: storage test detection technology and accelerated test life evaluation technology in normal environment. Storage test under normal circumstances testing technology, the test sample will be placed under normal stress in the natural 
environment for long-term storage, testing of its various performance changes, according to certain criteria, and then infer its storage life. The normal environmental storage testing technology needs to evaluate the adaptability of the sample stress environment and analyze the failure mechanism. Then, based on the analysis of the life test data, the service life prediction is made and the corresponding countermeasures are finally taken. Although the test cycle of normal environmental storage test is longer, the data is more practical and reliable [5].

Accelerated life test evaluation techniques is placing the sample at higher than normal ambient stress, obtaining the required reliability information through this accelerated environment, and using the associated physical failure pattern to derive the sample's life-time under normal stress. Accelerated test evaluation technology can significantly shorten the test time and effectively save resources, thus increasing the test efficiency.

\subsection{Overview of Foreign Research Status.}

At present, Russia and the United States are the world leaders in the life assessment of new types of ammunition. Many useful achievements have been made in the life prediction and reliability analysis of new types of ammunition. Normally, they have adopted normal environmental storage test and accelerated life expectancy Test evaluation method to evaluate the product storage life, and the test object gradually extended to various parts of the new ammunition components, such as propellants and propellants, combustible cartridge and optoelectronics and control components [6].

However, there are obvious differences in the technical approaches between Russia and the United States. The United States mainly adopts the method of natural storage test technology and supplemented by accelerated life test. Their main experience is the implementation of a storage reliability program from the delivery of new ammunition to monitor the aging trend of the product over the entire storage life; accelerated life testing technology as a new life prediction technology to infer the life of ammunition, 48-month service life prediction using accelerated aging technology [7]. The United States launched CD4011 "Actual Life Plan" and "Accelerated Life Plan" [8] to evaluate the operational performance of the smart ammunition stored by smart meters by functional monitoring, laboratory testing and flight testing provides data support for its life assessment. Such as "Dow antitank missiles," after a full range of functional testing, laboratory testing and flight testing, its life expectancy from the original 5 years to 22 years. Tests conducted through natural stockpile monitoring are generally true, because their storage environment conditions and test results are more credible, and it is independent of sample size limitations; however, it has longer test cycles and typically take years.

In contrast, Russia mainly uses accelerated life testing techniques to assess the shelf life of new types of ammunition, as well as supplementing and comparing data from natural storage tests. Its notable feature is a higher level of accelerated life test and can even reach the full level. The key to accelerated life test technology is improve environmental stress, and according to the aging of the physical model to establish the corresponding accelerated life model. There are many types of stress that affect material aging. In the Russian national standard, $\Gamma$ o $\mathrm{C}$ T 9.707, the dynamic processes of more than 20 stress types are described and a corresponding set of accelerated test software has been developed [9]. The accelerated life test software is based on the mathematical model for calculating the test status. To form a complete accelerated life test program, the following initial information needs to be input to the software: (1) storage environment condition related statistics (such as temperature and humidity Etc.); (2) storage locations; (3) sample bill of materials, including a detailed inventory of each material and its energy characteristics; (4) quantity, status and characteristics of each component of the sample, as well as its storage failure rate and reliable life expectancy[10].

Russian experts believe that accelerated life testing has the characteristics of a short test period. It can find out the weak points of ammunition in the product development stage, record the entire degradation process of the product, and further process the ammunition for improvement to deduce the inherent storage of ammunition life expectancy, and can simulate many environmental conditions of use area. As for the C-300 air defense missile, Russia conducted its accelerated life test for only 6 months and concluded that its storage life could last if 10 years [11]. Moreover, through accelerated 
life testing, the factory can make improvements based on the recorded weaknesses before mass production, to effectively avoid unnecessary waste after mass production and adjustment. However, limited to experimental techniques, conclusions derived from accelerated life tests are still quite different from the real ones and need to be compared with the results of natural environment storage.

However, at the same time, as the structure of informational ammunition system is more complicated, the accelerated life test is an area that accelerates the life test of complex systems. In control systems, there are usually multiple failure mechanisms, so system failure is the result of multiple potential failure mechanisms competing with each other. The problem of accelerating life test of complex systems under competitive failure conditions has attracted great attention of scholars both at home and abroad and the discussion of related issues will remain the focus of research for some time to come [12].

Although our country has gained some experience and theories in the assessment of the storage life of missiles, these research results cannot be completely applied to all new types of ammunition. Only by selecting targeted methods and conditions for different product categories can we draw conclusions relatively credible storage life, and then study its longevity repair technology.

\section{Accelerated Life Test Summary}

As the most commonly used testing technique for the assessment of complex equipment reliability, accelerated testing technique is an emerging engineering technique developed to assess the reliability of a product and used to improve product quality. Started in the 1950s, specialized research began in the 1960s. After the maturity of the theory of technology in the 1970s and further vertical and horizontal development in the 1980s, since the 1990s, accelerated testing techniques has entered a new phase of deeper and broader interdisciplinary new field and transformation to practical engineering.

\subsection{Concept Definition}

For the first time in 1967, the American Roma Air Development Center provided definitions of accelerated life testing: Accelerated Life Testing (ALT) relies on reliability theory and engineering practices to place products on reasonable acceleration models higher than the normal operating environment accelerated environment, thus obtaining the relevant reliability characteristics and its analysis inferred in order to reach the product under normal working stress stored life information of a trial[13].Accelerated life tests are in most cases applied to tests where the product failure mode is a burst failure mode.

\subsection{Accelerated Test Type}

Divided by the type of stress loading test can be divided into constant stress acceleration test (constant plus test), step plus stress acceleration test (step plus test), progressive stress acceleration test (sequence plus test) three, as shown in Figure 3 Show. For the above three kinds of accelerated test methods are described below:

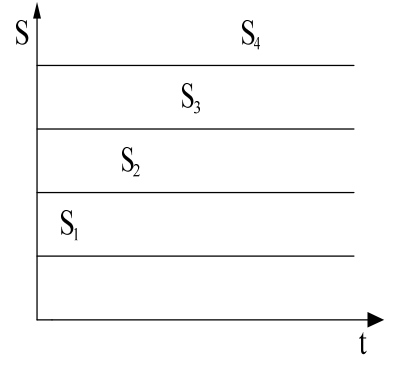

(a)

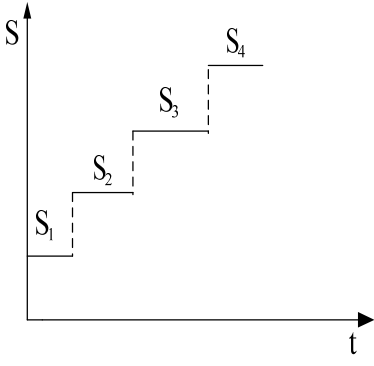

(b)

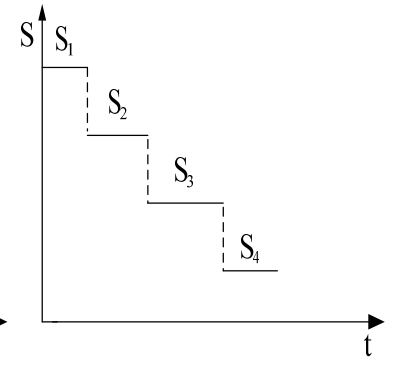

(c)

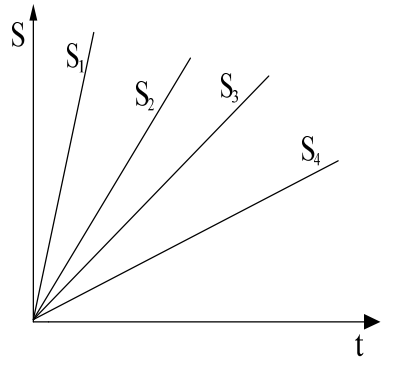

(d)
(a). constant plus test
(b). Step plus test
(c). Step down test
(d). Sequence plus test
Fig. 1Strss type of accelerated test 
1) Constant stress acceleration test $[14,15]$

Select a group of stress levels S1, S2, L, SK (S0, $<$ S1, $<$ L, $<$ SK) are higher than the normal stress level S0, the sample according to the number of stress levels assigned to each stress level were tested until the test censored conditions up to date.

2) Step plus stress acceleration test $[14,15]$

Select a group of stress levels S1, S2, L, SK (in a certain order of magnitude, you can gradually increase the step, can also be gradually reduced to step down) are higher than the normal stress level S0, first of all placed in the sample stress level S1 was tested Until a test tail-dead condition at that stress level is reached, change the stress level to S2, and continue testing the unedited specimen until the test-stop condition at that stress level is reached, repeating until the unexpectable specimen Sk is at creep conditions are reached at stress levels.

The step-plus test can be divided into step test and step-down test. Relevant foreign research results show that the step-down test can significantly shorten the test time for the products with long life and high reliability, which has higher efficiency and obvious advantages. This is because the walk-in test begins with loss of life from high stress, decreases stress level after failure, but since the sample has undergone high stress levels over a period, the entire test period will shorten the test time, so it can quickly and easily get the sample fault or reliability information.

Unlike constant tests, specimens in stepping tests tend to experience multiple stress levels, which gives them the advantage of being quicker and fewer in number.

3) Progressive stress acceleration test $[14,15]$

Select a set of stress levels $\left(S_{i}(t), i=1,2, \cdots k\right)$ as a function of time, generally select the time proportional to the stress level. Failure of the sample due to rapid changes in stress levels is also rapid.

The above three types of acceleration methods are applied in all countries, but the most common way of constant plus test, detection and evaluation techniques are also the most mature. Step Plus (including step and step down) test is currently in the research stage, it can improve the test efficiency, but also a small amount of applications. The lack of equipment and data analysis in the order-plus test makes it difficult to apply it to engineering [16]. For long-life, high-reliability products, in order to further shorten the test time and improve test efficiency to meet the needs of the actual project, accelerated test can be used in the form of step-by-step test [17].

\section{Inertia Gyro Assembly Storage Life Assessment}

\subsection{Thoroughly Test}

In the inertial gyroscope components for life assessment, if you want to use accelerated life test technology, you first need to conduct relevant products thoroughly test. The bottom of the test is usually placed in the sample higher than the normal environment under accelerated stress environment, through the test results to analyze the following points:

(1) Whether the sample is accelerable, whether the failure information of the product under pre-experimental accelerated stress is increased compared with that in the natural storage environment;

(2) Whether the failure mechanism of the specimen under high stress is consistent with the failure mechanism under the normal environment storage, whether the failure occurred during actual storage is reproduced in the accelerated test;

(3) In the case of failure mechanism has not changed, the performance of the sample test results is summarized, if no degenerative rules can be found, only the product of the failure time information, you can carry out accelerated life test;

(4) To determine the type of follow-up test, sensitive acceleration stress and stress level limit by analyzing the result of the experiment. 


\subsection{Formal Test}

Through the data analysis and reasonable judgments on the bottom of the test, you can determine whether accelerated life test method can be used to assess the reliability of the product. The basic steps of the research on reliability assessment based on accelerated life test as follows:

(1) Determine the method of accelerating stress application.

In general, the application of accelerated stress mainly takes the three types mentioned above, that is, constant stress method, step stress method, and progressive stress method. Usually we need to pre-accelerate the sample according to the specific purpose of the way to study to determine the application of stress.

(2) Determine the accelerated model of accelerated life test.

Acceleration model (also called acceleration equation): An equation used to express the relationship between life characteristics (such as median life expectancy, life expectancy and other general characteristics) and the stress level. The most commonly used acceleration tests are the following acceleration models [18]:

(1)Arrhenius model and single-stress Eyring model, with temperature as accelerating stress;

(2) inverse power law model, using electrical stress as accelerating stress;

(3) generalized Eyring model, using both temperature and voltage as accelerating stress;

(4) Multi-stress generalized Eyring model, usually have a number of stress as acceleration stress.

Different accelerating models correspond to different accelerating stresses, and the appropriate accelerating model needs to be determined based on the type of stress that affects the shelf-life of key components within the control system. In addition, the above acceleration model can only work within a certain range of stress, because failure of the sample will change as soon as the specified stress range is exceeded, and a new acceleration model must be established.

(3) determine the product life distribution.

In general, you can combine the sample's natural storage test data and accelerated test data to get their life distribution function. The most commonly used product life distribution mainly has the following distribution:

(1)Exponential distribution, the most common life distribution, can be used to describe the life of some electronic components and has one important property - no memory, that is, the products subject to exponential distribution are in use for a period. The remaining life after the same with the new life expectancy to follow the original index distribution;

(2) Weibull distribution is a widely used continuous distribution in reliability that can be used to describe life distributions such as fatigue failure, vacuum tube failure, and bearing failure;

(3) Lognormal distribution, products such as insulators, diodes generally obey this lifetime distribution.

(4) determine the life expectancy method.

Statistical methods such as point estimation, interval estimation, moment estimation, maximum likelihood estimation, least-squares estimation and Bayesian estimation can usually be used when processing truncated life-span data or full-life data for accelerated storage life testing. In contrast, maximum likelihood estimation can better integrate all kinds of information in the sample experiment, and the calculation is relatively simple, while the accuracy is not too bad [19]. In the life-span estimation method with relatively small number of samples, the shrinkage estimation of fusion prior information has better statistics than the maximum likelihood estimation [20].

\section{Conclusion}

Foreign countries have carried out more studies on the life assessment of information-based ammunition. The European and American countries and Russia have been able to achieve system-level life assessment. The life expectancy and reliability analysis applied to new types of ammunition and weaponry have achieved many relatively mature results. In contrast, our country is still in the stage of learning and initial exploration in accelerating life testing: the research on 
accelerating life testing in our country is short. In addition to the technical blockade in other countries, there is still a huge gap between developed countries on software and hardware. At present, there is no practical design method for accelerating the life test of the key components of informational ammunition. In the case of the accelerated tests of subsystems of the informational ammunition storage life span and even the whole bombs, there is an urgent need to deepen the study of relevant theoretical systems and to improve the technical means of accelerating life testing.

\section{References}

[1]. Wang Dongmei, Dai Wenjang, Zhang Yongtao. Research Status and Development Trend of Informational Ammunition [J]. Acta Phys Sinolica Sinica, 2010, (32).

[2]. Ma Jing,Lu Rongcui,Li Xiaoyang. Reliability Analysis of Sensor component based on fault tree analysis [J] .Journal of Chinese Inertial Technology, 2008, 16 (4): 65-70.

[3]. Zhang Shengpeng, Wang Xiaohong, Li Xiaogang. Design of experimental scheme for accelerated storage of electronic machines [J] .Quality and Reliability, 2011,152 (2): 24-28.

[4]. Luo Tianyuan, Zhou Kun, Yu Shuhua, Zhu Lei. A Summary of Test and Evaluation Techniques for Storage Life of Ammunition [J] .Equipment Environmental Engineering, 2005,4: 17-22

[5]. Niu Yue listen, Mu Xihui. Prospects for the evaluation of storage life of informational ammunition [J] .Equipment Environmental Engineering, 2013,5: 94-97,101

[6]. Wang Yong-nan, Mu Xi-hui, Niu Yue-sheng, Du Feng-po, Luo Jian, Chen Jian-hua.Analysis of Reliability Evaluation of Missile Control System Based on Information Fusion [J]2015,7:71-74

[7]. Li Haibo, Zhang Zhengping, Hu Yanping. Accelerated life test method and its application in aerospace products [J]. Intensity and Environment, 2007,1: 2-10

[8]. Joseph Martinelli Pecht Associates. Effect of Long-Term Storage on Electronic Devices [R]. U.S. Army Armament Reserch, Development and Engineering Center, 1995.11.

[9]. Zhang Hong Hou Xijiu. Russia accelerated storage and accelerated transport test technology overview [J]. Study abroad technical report, 1997, 2: 109-113.

[10]. Chen Bing, Li Xing.Study on Engineering Application of Accelerated Life Test Technology at Home and Abroad [J]. Intensity and Environment, 2010,6: 31-38

[11]. Feng Zhigang, Fang Changhua, Li Jing.Analysis of Accelerated Aging Tests of Foreign Missiles [J]. Chinese Journal of Missiles and Space Transportation, 2008,2: 30-34

[12]. T.A. Mazzuchi C. Bunea. Competing failure modes in accelerated life testing [J]. Journal of Statistical Planning and Inference, 2006, 136: 1608-1620.

[13]. Shu Xiumei, Zhang Shifu, Liu Tianmin, Luo Yuanyuan. Review of Accelerated Life Test [J]. Journal of Logistical Engineering University, 2012,6: 45-50

[14]. Wang Lingling Shimatsu. Accelerated life test [M]. Beijing: Science Press, 2000.

[15]. Chen Xun, Zhang Chunhua, Wang Yanshun, Tan Yuanyuan. Accelerated life test technology and application [M]. Beijing: National Defense Industry Press, 2013.

[16]. Tan Yuanyuan. Equipment storage life of accelerated testing technology [D]. Changsha: National University of Defense Technology, 2010.

[17]. GAO Meng, WANG Jinzhu, HE Xueguang.Study on storage life of guided munitions based on reliability statistical analysis [J] .Equipment Environmental Engineering, 2013, 10 (3): 110-113. 
[18]. Song Ya-nan, Li Xin-jun, Liu Xue-feng, Zhang Shi-nian, Wang Shizhen.A review of optimization design for accelerated storage life test $[\mathrm{J}]$.Electronic Product Reliability and Environmental Test, 2009,21: 189-192

[19]. Xu Tingxue, Wang Haowei, Zhang Xin. Reliability Modeling Method Based on Gamma Process in Step Acceleration Test [J]. Modern Defense Technology, 2015,1: 124-128,134

[20]. Hong Dong-run, Zhao Yu, Ma Xiao-bing .Shock estimation of generalized Weibull distribution parameters [J] .Acta Aeronautica Sinica, 2009,6: 2442-2446 